\title{
Fractal Logic Applied to Micro-grained Textures Characterization
}

\author{
Giuseppe Bonifazi, Andrea D’Addetta
}

Dipartimento di Ingegneria Chimica, dei Materiali, delle Materie Prime e Metallurgia, Università degli Studi di Roma "La Sapienza", Via Eudossiana, 18, 00184 Rome, Italy, Telephone: +39.06.44585.925 Fax:+39.06.44585.618 e-mail: giuseppe.bonifazi@uniroma1.it

Micro-grained textures numerical characterization and modelling represent one of the most challenging topic of computer assisted microscopy. Its relevance affects many research and industrial sectors ranging from microbiology, to medicine, from materials and environmental science, to chemical, pharmaceutical and food industry, etc. . Such a characterization can be reached following different strategies: i) adopting a classical human based approach, or ii) following a more fully digital-imaging approach, that is to try to extract parameters more linked to the intrinsic characteristics of the images and not strictly related to human expert direct evaluation. The approach described in the following, even if dating back in terms of logic and algorithms to the early 80's [1], follows this second strategy and it is based on the, "a-priori" assumption that a micro-grained texture can be considered, the repetition of one or more basic structures. In this perspective it was assumed that fractal geometry can adequately describe them [2]. Fractal geometry, formalised by Mandelbrot [1], concerns the study of non-Euclidean geometrical figures generated by a recursive sequence of mathematical operations. These figures show self-similar features in the sense that their shape, at a certain scale, is equal to, or at least "similar", to the same shape of the figure at a different scale or resolution. As for Euclidean figures a comparison of geometrical entities and the result of physical events can provide a characterisation of the underlying nature of the event. In particular the $2 D$ fractal approach described in this work allowed to quantify the textural attributes of micro-grained image fields through the evaluation of the recursiveness properties of the basic structures, characterized by specific morphological and morphometrical attributes and chemical/physical properties. Such an evaluation was carried out implementing the Sierpinski carpet approach [3], that is designing an analytical tool able to study bounded two dimensional space filling problems (Figure 1). The image is decomposed (segmented) into the domains defining the micro-granular texture. A certain number of dimensional classes for the domains are defined. The domains are then added again starting from those belonging to the largest classes up to the smallest. The rate of disappearance of the image background is computed. Finally, the Richardson plot is drawn representing, on a log-log plane, for every dimensional class of the domains, contributing to define the fine-grained texture, the corresponding residual background area (Figure 2). The described approach was successfully applied: i) to characterise micro-grained textures generated by stone airborne dust particles, produced by specific mechanical actions (Figure 3), when collected on a surface inside a duct [4] and ii) to identify the correlation existing among materials, operations and tools responsible of dust production, to improve working environmental conditions trhough optimal caption systems and filtering architecture design [4].

[1] B. B. Mandelbrot, The Fractal Geometry of Nature, Freeman, San Francisco, 1983.

[2] B.H. Kaye, A Random Walk Through Fractal Dimensions, VCH Publishers, Weinhein, 1989.

[3] B. B. Mandelbrot, Self-Affine Fractal Sets, Fractals in Physics, North-Holland, (1986) 3-28.

[4] G.Bonifazi et al., On-line 2D Fractal Based Techniques to Characterise Respirable Dusts. International Congress on Particle Technology: PARTEC 2001, Nurnberg, Germany, 2001. 

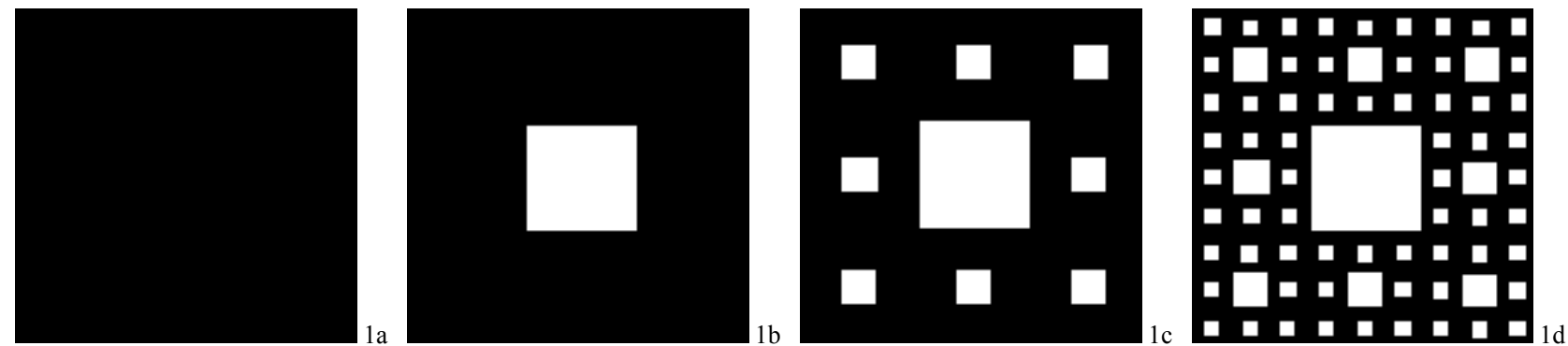

Figure 1 - The constructor algorithms of the progressions displayed are composed of an initiator (1a) and a generator (1b). 1c: first and 1d: second order iterations. The initiator is a filled square. The initial side of the initiator, has been divided into segments of equal interval according to the ratio of similarity $\mathbf{r}$, which is $1 / 3$. The total number, $\mathbf{N}$, of the remaining un-removed squares in the generator is 8 . The Sierpinski fractal dimension $\mathbf{D}$ of the system can be determined using the standard equation to compute fractals, $\mathbf{D}=\log \mathbf{N} / \log (1 / \mathbf{r})$ and yields a value of 1.8928 .
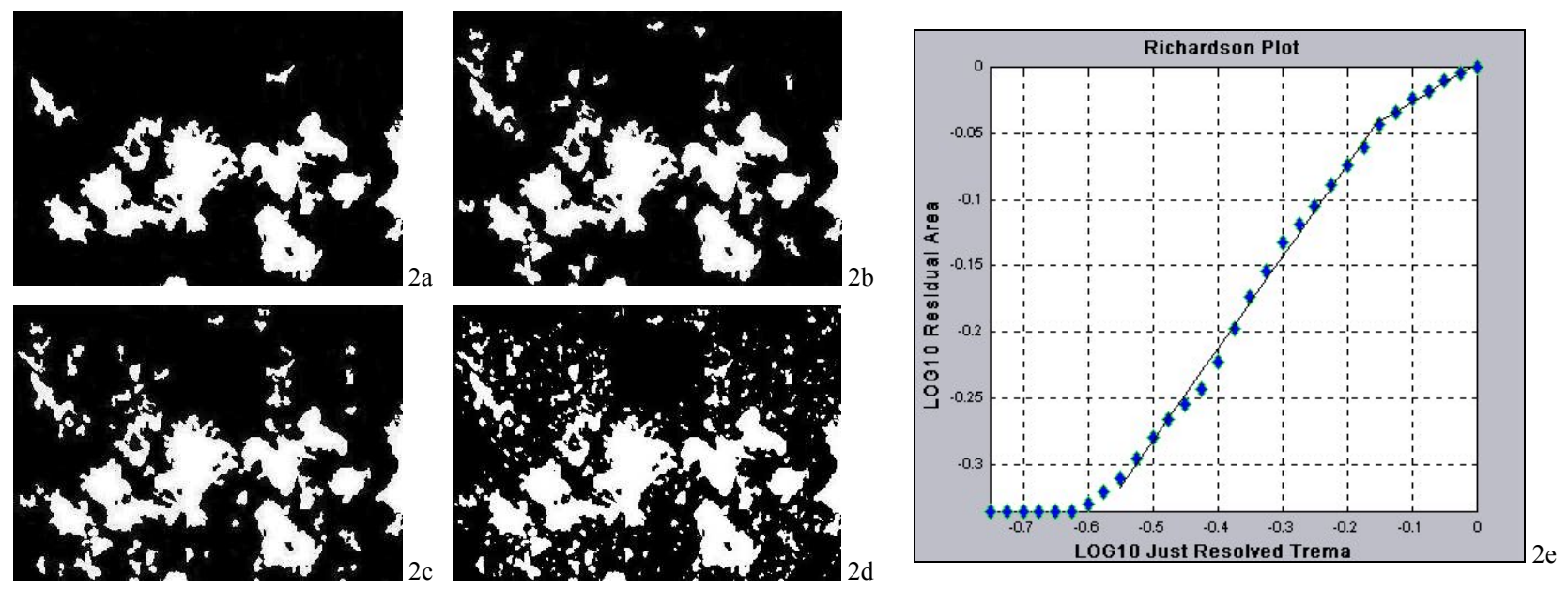

Figure 2 - The algorithm (from 2a to 2d) to compute fractal is shown. 2e: Richardson plot. The values on the $\mathrm{x}$-axis of the plot are computed as square root of pixels area normalised dividing by the area of the largest particle (trema).

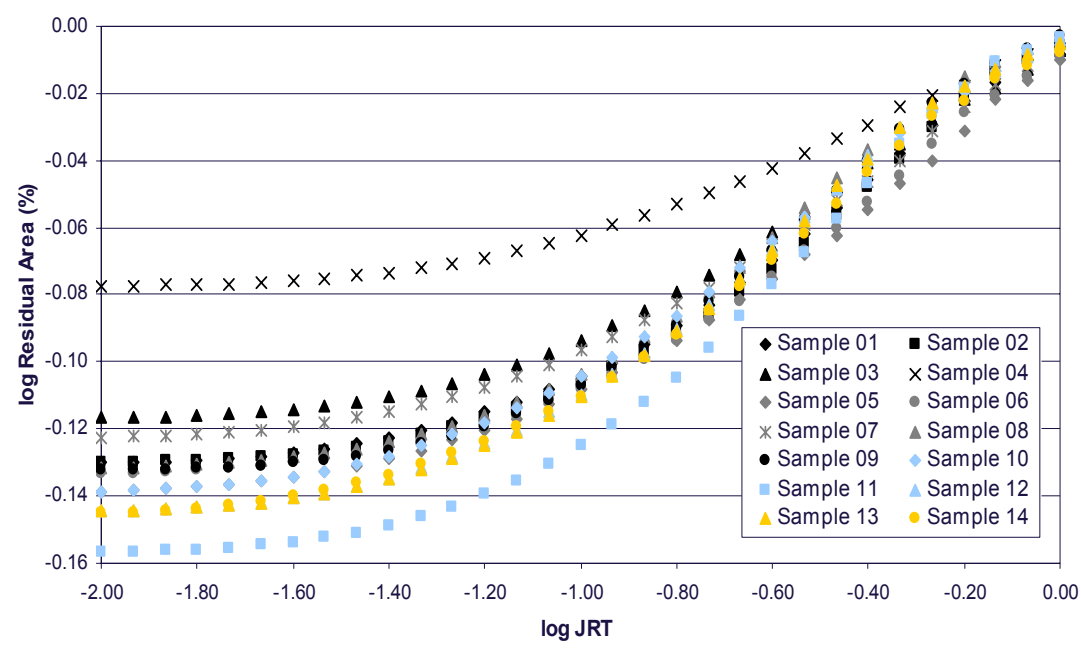

Figure 3 - Richardson Plot of different powders resulting from the autogenous milling of 14 different stones. The plot clearly shows the different behaviour of the materials according to their intrinsic characteristics.

Sample 1: Travertino, Sample 2: Rosso Verona, Sample 3: Botticino, Sample 4: Bianco Carrara, Sample 5: Thassos,

Sample 6: Verde Guatemala, Sample 7: Nero Marquina, Sample 8: Grigio Sardo, Sample 9: Nero Assoluto, Sample 10:

.00 Labrador, Sample 11: Rosso Multicolor, Sample 12: Basaltina, Sample 13: Pietra Dorata, Sample 14: Pietra Serena. 
